\title{
Parameterization Of A Simplified Physical Battery Model
}

\author{
Grimm Alexander $^{1}$ Prof. Haumer Anton ${ }^{1}$ \\ ${ }^{1}$ Ostbayerische Technische Hochschule Regensburg, Germany alexander.grimmest . oth-regensburg. de, \\ anton.haumerdoth-regensburg.de
}

\begin{abstract}
The importance of batteries is increasing, especially in the field of the high power requirement systems like electric driven vehicles. Mobile energy storage makes it possible to accelerate with incredible torque, without any accruing air pollution. Due to the high costs of real components, it is of great use to simulate battery driven systems before building them. Transient processes within a cell are highly dependent on the operating point of the complete system, which makes it difficult to create equations and model parameterizations. This paper shows which data is important for cell modeling and how to parameterize simplified physical cell models.

Keywords: simplified battery model, battery parameterization, physical battery model
\end{abstract}

\section{Introduction}

The main target of the master student's project was to get a method to generate a simplified model of a lithium ion cell. In general the physical models of batteries are generated with electronic elements like resistors, capacitors, or inductances.

The complexity of battery models can go up to infinity, so it is necessary to simplify the structure as much as possible. The simpler models contain a resistor, which represents the inner resistance of the cell and a resistor connected in parallel with a capacitor, which represents the capacitive behavior. Because of that, this model can only recreate the real battery behavior in a few situations and is also the least accurate one. To generate a more precise simulation, it is necessary to use more RC-elements (resistance connected in parallel with a capacitance). With more of these elements, it is possible to fit the Nyquist plot of batteries much more accurately.

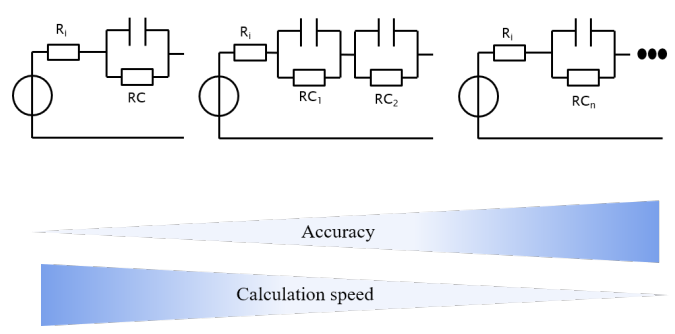

Figure 1. Rising calculation speed versus higher complexity

For high frequencies the impedance goes even inductive, so therefore a inductance should also be considered.
The disadvantage of more elements in the equivalent circuit is that more information about the cell is needed for parameterization and the CPU time for calculations is increased.

The most accurate models are completely numeric models in which all Elements are parametrized with interpolated tables, which are depending on temperature, SOC (state of charge) and flowing current. These models need much more measurement data than the ones with simple concentrated elements. All the parameters have to be recalculated in every state of the battery, which causes an extreme rise of the calculation time for the simulation. The measurements are very time consuming and expensive, so not many institutions have access to the necessary information and data for this kind of simulation models. That is the reason why in this paper no such model is discussed and presented.

\section{Characteristic curves}

\subsection{Open circuit voltage}

The open circuit voltage (short OCV) describes the cell voltage without any load, depending on the state of charge. With lower SOC the voltage of the cell is lower and vice versa. Mostly the OCV gets measured between SOC $<95 \%$ and SOC $>5 \%$, so the cell does not get overloaded, or completely discharged, because both scenarios would harm the cell irreversibly. To get the information for the behavior higher, or lower than the measured values, the graph can be extrapolated.

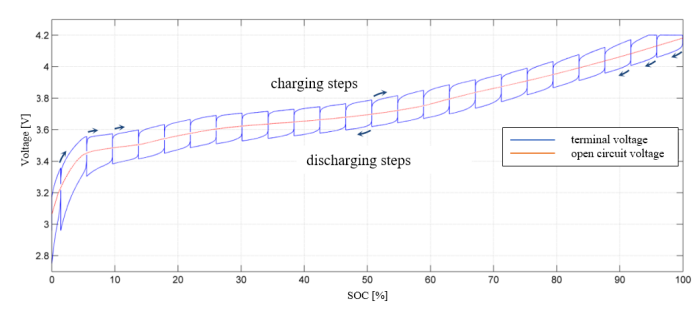

Figure 2. OCV of a battery with dis/charging steps (Peter Keil, p.3)

The OCV calculation is based on a charging and discharging process within the cell, where it gets charged/discharged with constant current impulses. The mean value out of both curves is the OCV. 

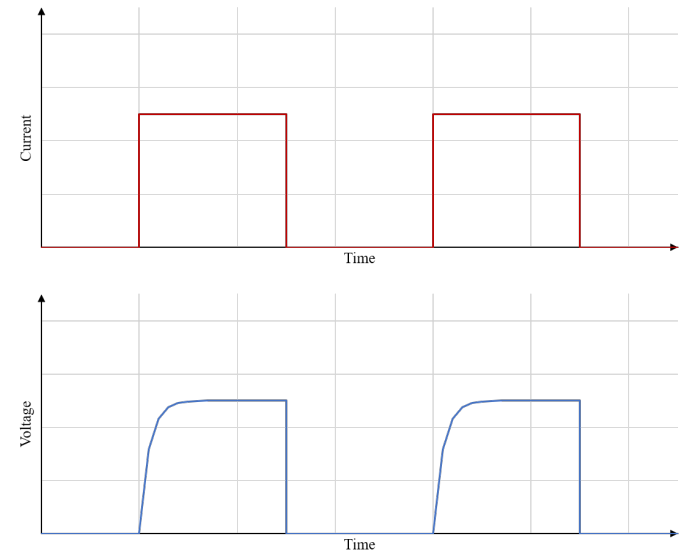

Figure 3. Schematic representation of a battery impulse response

\subsection{Impulse response}

The Impulse response shows the dynamic behaviour of the cell. To measure this specific curve, the battery gets electrically powered with predefined current impulses. It is possible to read a general time constant out of the graph for parametrization (Figure 3).

\subsection{Electrochemical impedance spectroscopy}

The electrochemical impedance spectroscopy (short EIS) shows the cell impedance depending on the current frequency and temperature. It is currently the most accurate method to describe the battery impedance behavior, but also the most elaborate and expensive with regards to the costs for measurements. Every single graph shows the behavior of the impedance for a fixed temperature and SOC. That means, that the EIS graph is different for every SOC and temperature.

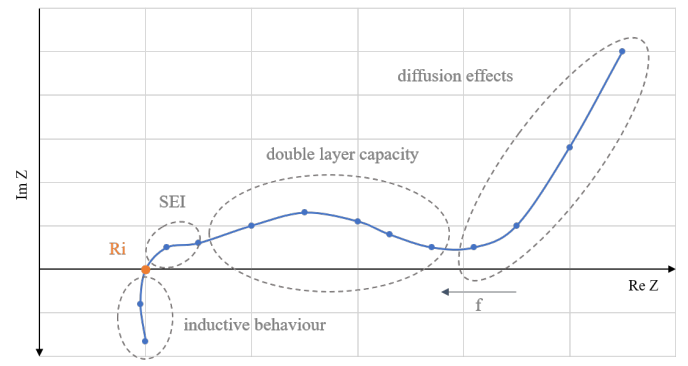

Figure 4. Schematic representation of an EIS

Figure 4 shows the graph of an EIS, which begins on the right hand side with $f \approx 0 \mathrm{~Hz}$ and continue to the left with rising frequency. It can be divided into four sections, which show an individual characteristic of the cell. By low frequencies, the effect of the diffusion of the chemical ingredients influence the behavior the most. When the graph rises again, the effect of the double layer capacity, which is caused by the structure of a lithium ion cell, has the most impact. The section between the crossing point with the real part axis and the section for the double layer capacity shows the influence of the solid electrolyte interface on the impedance. The crossing point represents the inner resistance of the battery. When the frequency rise above this point, the battery begins to show inductive values, which can be simulated by inductances. It is important to know in which operating point the simulation will run mostly, so different equivalent circuits can be chosen for better results.

\section{Parametrisation}

There are many different models with different possibilities to parameterize them, but all of them have in common that they need the open circuit voltage for the basic voltage supply behavior. The data of the OCV can be interpolated and used for a voltage supply. In Modelica, there can be used a signal voltage and a CombiTable2D element and both are given in the Modelica Standard Library. The CombiTable2D can read the table of the OCV and provides the corresponding signal to the signal voltage source. If more OCV measurements within different temperatures are given, it is also possible to interpolate between the tables and provide a continuous behavior of the $\mathrm{OCV}$ within the temperature range. If the temperature can be seen as constant and it is precise enough for the individual application even data with different temperature, is not necessary.

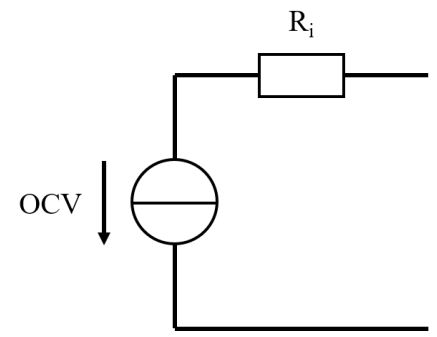

Figure 5. Equivalent circuit with inner resistance

The inner resistance can be calculated based on the voltage drop, which can be seen between the OCV and the voltage while charging/discharging the cell (figure 2).

$$
R_{i}=\frac{\Delta U}{I_{\text {step }}}
$$

\subsection{Battery model with one RC-Element}

There are two options to parameterize this model. It is possible to use the parameterization with the pulse response or with the electrochemical impedance spectroscopy. To do so with the pulse response is the much easier one and it does not need very expensive measurement systems like the other method. But like all other following models, it needs the OCV like the first and simplest model "Battery model with $R_{i}$ ".

The impulse response of a battery cell shows the inner resistance with the current and voltage in the first moments of the impulse. So the value of $R_{i}$ (inner resistance) 
can be calculated just by dividing voltage through the current. With the stationary current, that occurs after a short time $(\Delta I \approx 0)$ the resistance of the RC-element can be calculated by:

$$
R_{R C}=\frac{U}{I_{\text {stat }}}-R_{i}
$$

With the information about $R_{R C}$ and the impulse response, the capacitance can be calculated with the time constant $\tau$ of the rising current. $\tau$ needs to be read out of the impulse response.

$$
C=\frac{\tau}{R_{R C}}
$$

The inner Resistance can be again calculated with the OCV:

$$
R_{i}=\frac{\Delta U}{I_{\text {step }}}
$$

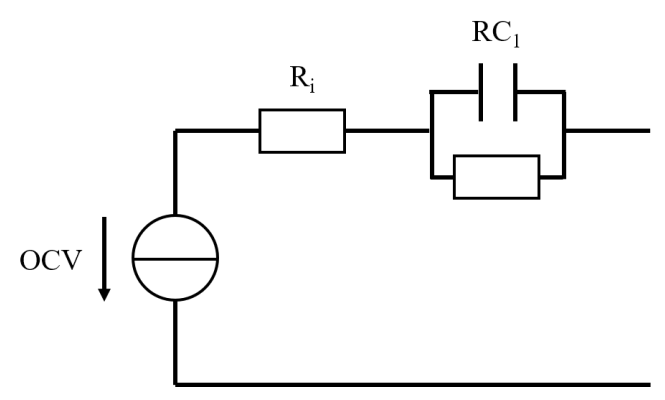

Figure 6. Equivalent circuit with one RC-Element

\subsection{Parametrisation of any Model with the EIS}

For this, the electrochemical impedance spectroscopy (short EIS = Nyquist plot of a battery) of lithium cells has to be analysed and evaluated. To use this method for parametrization correctly, it is important to know in which frequency band the simulation will run. As said in 2.3 the EIS itself shows the impedance depending on the frequency of the battery and it can be divided into four sections, which show the individual characteristic of the elements of the equivalent circuit.That means, that the individual segments of the curve can be used to identify the parameters for the significant elements, which should represent this characteristic in the equivalent circuit. Every individual section of the EIS can be fitted with an algorithm and the formula for the impedance for each element. Therefore optimization tools like the curve fitting toolbox, optimization toolbox for Matlab and the Solver for Microsoft Excel can be used. The following example shows how it is done.

For this example the equivalent circuit with inner resistance and two RC-Elements is chosen. This structure is a very common model for batteries and it should provide very high accuracy for low level frequencies (frequencies in which the battery behavior is not inductive). For the battery model application in cars, it can be said, that speed requirement (pedal position) does not change with high frequencies $f \approx k H z$ and so doesn't the current for the motor with additional loads.

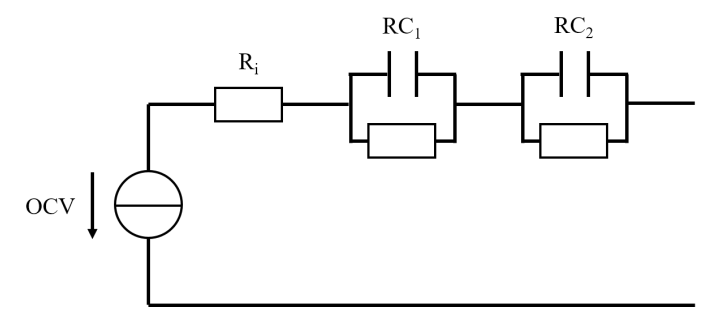

Figure 7. Equivalent circuit with two RC-Elements

To get the parameters for the other elements, it is necessary to know how the impedance for the structure is calculated. With the knowledge about the formula for the impedance it is possible to use a fitting algorithm to calculate the capacitances for the capacitors and the resistances for the resistors.

$$
\begin{gathered}
Z_{G}=R_{i}+Z_{R C 1}+Z_{R C 2} \\
Z_{R C 1}=\frac{R_{1}}{\left(\omega R_{1} C_{1}\right)^{2}+1}-\frac{j \omega R_{1}^{2} C_{1}}{\left(\omega R_{1} C_{1}\right)^{2}+1} \\
Z_{R C 2}=\frac{R_{2}}{\left(\omega R_{2} C_{2}\right)^{2}+1}-\frac{j \omega R_{2}^{2} C_{2}}{\left(\omega R_{2} C_{2}\right)^{2}+1}
\end{gathered}
$$

This leads to the separated real and imaginary part of $Z_{G}$ :

$$
\begin{gathered}
R_{G}=R_{i}+\frac{R_{1}}{\left(\omega R_{1} C_{1}\right)^{2}+1}+\frac{R_{2}}{\left(\omega R_{2} C_{2}\right)^{2}+1} \\
X_{G}=-\frac{j \omega R_{1}^{2} C_{1}}{\left(\omega R_{1} C_{1}\right)^{2}+1}-\frac{j \omega R_{2}^{2} C_{2}}{\left(\omega R_{2} C_{2}\right)^{2}+1}
\end{gathered}
$$

The next part of the parameterization of a battery model with the EIS is to look up the measurement table of the EIS and choose the suitable data for the recreation of the graph. If the model has no inductance included, it is not possible to simulate inductive behavior. So all data with positive imaginary part is not suitable for the fitting and can be ignored.

The next step is to use the formula for the resistance (eq.:8) and the reactance (eq.:9) and minimize the deviation of the error squares:

$$
\begin{aligned}
& \left(R_{\text {measured }}-R_{\text {calculated }}\right)^{2} \stackrel{!}{=} \min \\
& \left(X_{\text {measured }}-X_{\text {calculated }}\right)^{2} \stackrel{!}{=} \text { min }
\end{aligned}
$$


Now it is time to use the fitting tool to calculate the four remaining parameters for the RC-Elements. The tool should find the minimum of the error squares by changing the parameters for the RC-Elements. Every solving algorithm needs well chosen start values for the calculation. Therefore the resistances should not be higher than realistic values. The resistances are commonly not higher than one $\mathrm{Ohm}$ for li-ion battery cells. The capacitances are more difficult to select. Every cell chemistry is different, even if it also is among li-ion batteries and therefore the capacitances vary massively. If there aren't many informations about the cells and the start value available, zero Farad can be a good value for the beginning of the fitting process.

The solving algorithm tries to find the minimum for the error squares by changing the values of the RC-Elements and the formulas for the impedance. All of those algorithms are iterative solving methods which search for local minimums of the given functions and parameters. With the wrong start values the results will be false and need to be evaluated. This needs to be kept in mind.

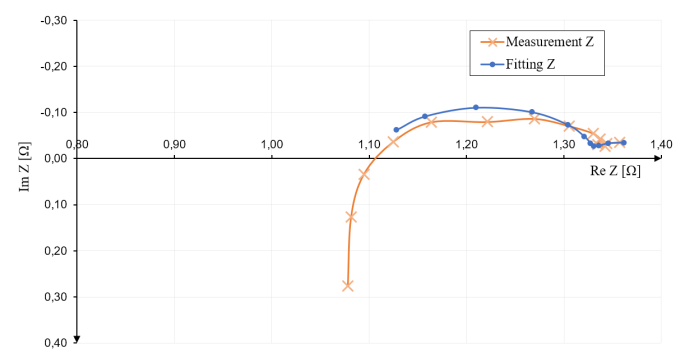

Figure 8. Fitting result with example measurement data.

Figure 8 shows an example with the resulting impedances for the given frequencies. The fitting can approximate the measured graph very closely, which means, that the behavior of the real cell can be reproduced very well.

\section{Battery Model for long term energy investigations of electric vehicles}

This project "finding a suitable battery model and the possibility to parametrize it", is based on the previous project to create a Modelica based simulation library for modeling and simulation of complete electrically driven vehicles. To keep it simple and reasonable for a electical physical model, the following considerations were made.

\subsection{Chosen model}

After intense research in papers and books the decision was to take the cell model with inner resistance and two RC-Elements to get high accuracy and precision for the simulation. The structure of the RC-Elements can be seen as the two transition layers of the electrodes and the inner resistance like shown in figure 10. The two RCElements have to be parametrized differently, because of the different chemical structure at each represented transition layer. The Resistors $R_{i 1}$ and $R_{i 2}$ represent the electrical resistance of the electrodes themselves. $R_{e l}$ reproduces the resistance of the electrolyte for the lithium-ions to go through.

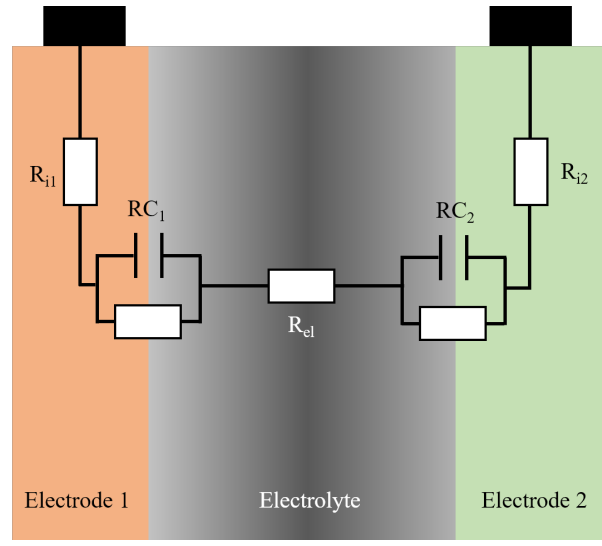

Figure 9. Consideration of the equivalent circuit.

The resistances $R_{i 1}, R_{i 2}, R_{e l}$ in sum result into $R_{i}$.

$$
\begin{aligned}
Z_{G} & =R_{i 1}+R_{i 2}+R_{e l}+Z_{R C 1}+Z_{R C 2} \\
& =R_{i}+Z_{R C 1}+Z_{R C 2}
\end{aligned}
$$

The model in the library needs additional elements for handling all the data and inclusion of the option to use all resistances for thermal management. An integrator converts the measured current into charge for calculation of the SOC. The SOC itself needs to be denormalized for the interpolation table of the OCV and all relevant signals are combined in the battery bus for a better overview. For a more realistic behaviour, the self discharge resistance $R_{s d}$ is also considered and set by default to $1 M \Omega$.

\subsection{Simulation and modelling}

Until now, this research project has no real EISmeasurements of cells from high voltage battery packs available. So it would make no sense to parameterize an model example of a car and try to simulate it, because it cannot be verified with any real measurements. The given graph in figure 8 at $p .4$ shows a value for the inner resistance that is way too high. With that data it is not possible to reproduce driving cycles with high acceleration and power requirements above $\approx 150 \mathrm{~kW}$. Nevertheless the EMOTH battery model was tested with fictive values and was completely operational. It has to be kept in mind that the simulation will abort with a singularity error if the resistance is to high for the power requirement and the data for the OCV can only interpolate between the given data and not be extrapolated. If the temperature is rising above the maximum given value or falling below the minim given value, it will abort the simulation. 


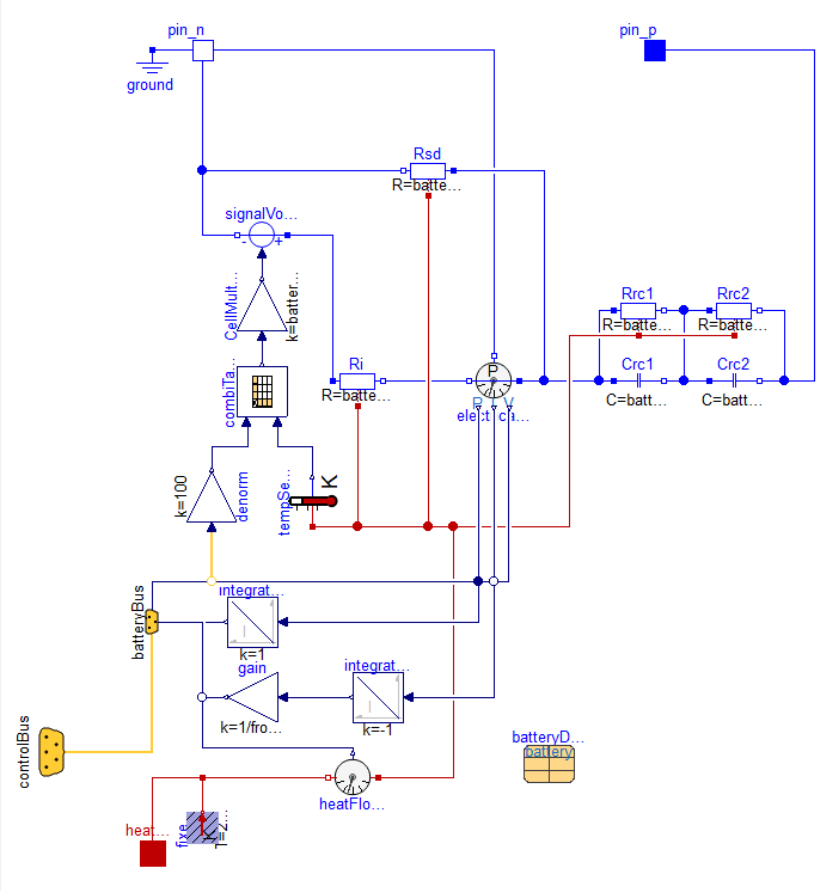

Figure 10. Equivalent circuit of the battery in Dymola.

For testing the functionality and the containing Modelicaelements, a simpler model was built and tested with different temperatures. The OCV was analysed and the results are shown in figure 11. The table2D interpolation was set to interpolate with continuous derivatives to smooth the graph. The parameters for the elements were set to the values obtained out of the measured EIS. Figure 11

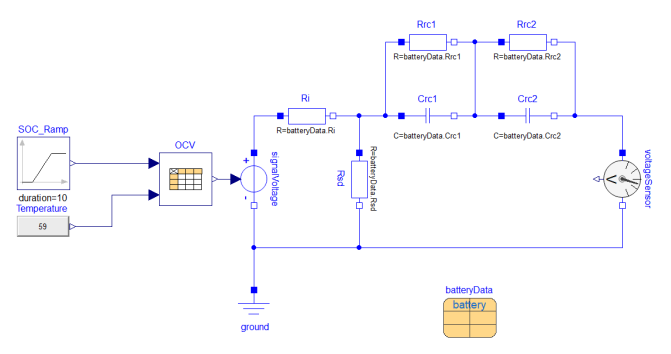

Figure 11. Battery model test for OCV behaviour

shows the structure of the test model. The SOC_ Ramp changes the SOC from $100 \%$ to $10 \%$ in 10 seconds with the fixed and predefined temperature, which is defined in the temperature block. All equivalent circuit parameters are summed up in the data record "batteryData". The voltage sensor measures the open circuit voltage depending on the changing SOC.

The graph in figure 12 shows the resulting interpolation from the example data from figure 8 p. 4 for two different temperatures $\left(T_{1}=-20^{\circ} \mathrm{C}\right.$ and $\left.T_{2}=59^{\circ} \mathrm{C}\right)$. If there are real and useful electrochemical impedance spectroscopies available with different temperatures and state of charges, the previous discussed models and parametrization meth- ods should provide very good simulation structures for accurate battery simulations.

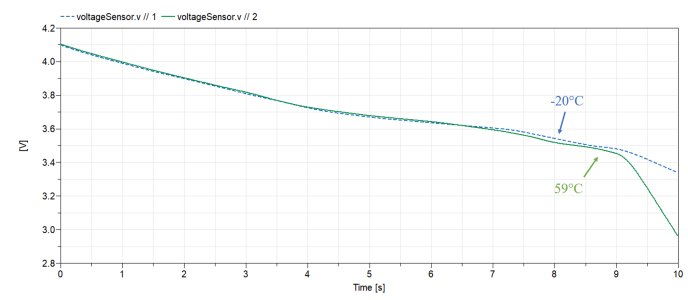

Figure 12. Recreated OCV in Dymola with different temperatures

\section{Conclusion and outlook}

There are many different ways to create a battery model, but the higher the level of complexity, the more calculation time and measurements are needed to be performed as basis for parametrization. Since this is very expensive and time consuming, the requirement of modeling is to keep the simulation structures as simple as possible.

In (Peter Keil) it is proven that even the equivalent circuit with only one RC-element can provide very accurate results. They used a model build up of one RC-Element and a predefined load profile for discharging the battery. Between the simulation and real measurements the difference was less than two percent, except for the operating points with a SOC lower than five percent, where the difference came up to $10 \%$. The choice for the battery model depends on the type of measurements that can be proceeded and the available calculation power.

The next step is to use the obtained results and combine them with additional data to create and parametrize a complete model of the electrically driven bus of Regensburg.

\section{References}

Peter Fritzson. Principles of object oriented modeling and simulation with Modelica 3.3. John Wiley and Sons Inc., 2015. ISBN 978-1-1188-5897-4.

Rodrigo Garcia-ValleJoao A. Pecas Lopes. Electric Vehicle Integration into Modern Power Networks. Springer, New York, NY, 2013. ISBN 978-1-4614-0134-6.

Wolfgang Mielke. Modellierung von Kennlinien, Impedanzspektren und thermischem Verhalten einer LithiumEisenphosphat-Batterie. PhD thesis, 2011.

Andreas Jossen Peter Keil. Aufbau und Parametrierung von Batteriemodellen. $\mathrm{PhD}$ thesis.

Otto K. Dietleier Peter Kurzweil. Elektrochemische Speicher. Springer Fachmedien Wiesbaden GmbH, 2015. ISBN 978-3658-10899-1.

D. V. Ragone. Review of battery systems for electrically powered vehicles. 1968.

Jan Philipp Schmidt. Verfahren zur Charakterisierung und Modellierung von Lithium-Ionen Zellen. PhD thesis, 2013. 
